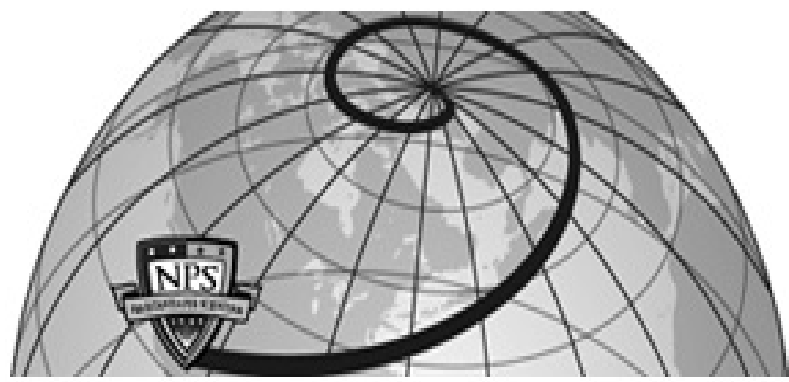

Calhoun: The NPS Institutional Archive DSpace Repository

1992

\title{
Autonomous Sonar Classification Using Expert Systems
}

Brutzman, Donald P.; Compton, Mark A.; Kanayama, Yutaka

https://hdl.handle.net/10945/41289

This publication is a work of the U.S. Government as defined in Title 17, United States Code, Section 101. Copyright protection is not available for this work in the United States.

Downloaded from NPS Archive: Calhoun

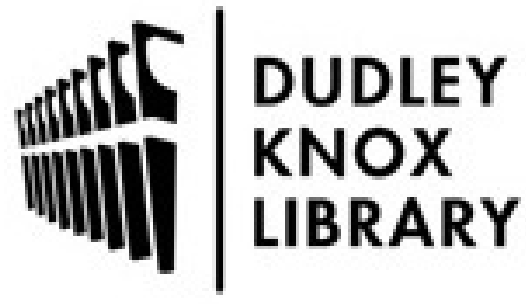

http://www.nps.edu/library
Calhoun is the Naval Postgraduate School's public access digital repository for research materials and institutional publications created by the NPS community. Calhoun is named for Professor of Mathematics Guy K. Calhoun, NPS's first appointed -- and published -- scholarly author.

Dudley Knox Library / Naval Postgraduate School 411 Dyer Road / 1 University Circle Monterey, California USA 93943 


\title{
AUTONOMOUS SONAR CLASSIFICATION USING EXPERT SYSTEMS
}

\author{
Donald P. Brutzman, Mark A. Compton and Yutaka Kanayama \\ Computer Science Department, Naval Postgraduate School \\ Monterey California 93943-5000 USA \\ brutzman@cs.nps.navy.mil \\ (408) 646-2149 work, (408) 646-2595 fax
}

\begin{abstract}
An expert system can process active sonar returns, perform geometric analysis and autonomously classify detected underwater objects. Autonomous classification of objects is an essential requirement for independent operation by autonomous underwater vehicles (AUVs). Most AUVs are only capable of rudimentary sensor analysis, since standard approaches to evaluation and classification of sonar data require excessive signal processing and computational power to be practical. This paper describes how to develop an autonomous sonar classification expert system for a working AUV.

A fundamental approach is presented for applying geometric reasoning and expert system heuristics to sonar classification. Preliminary sonar processing is performed using parametric regression line fitting. A polyhedron-building algorithm correlates the parametric regression line segments into geometric objects. After quantifying geometric object attributes, objects are classified using rule-based evaluation of quantitative and qualitative attributes combined with sonar classification heuristics. Real-time graphic simulation and scientific visualization are employed to evaluate results. Experimental classification results are presented using actual mission data from the Naval Postgraduate School (NPS) AUV. Successful classifications of walls and a mine-like object are demonstrated. I. INTRODUCTION
\end{abstract}

Intelligent vehicles will play a major role in future underwater missions. A critical requirement for independent behavior by such vehicles is autonomous analysis of complex and variable ocean environments. This is a notoriously difficult task, even when human operators use sophisticated sensors and powerful processors.

Although much work has been done in vision processing for mobile robots, additional research is needed on interpretation of observed scenes and terrain [1]. Numerous approaches to the general object-recognition problem are presented in [2]. Both of these references can be found in [3], an essential collection of surveys, tutorials and fundamental research papers regarding mobile robot sensor perception, mapping and navigation. Other pertinent references included in [3] are [4] and [5].

Independent and meaningful interpretation of sensor data is a principal prerequisite for accomplishing high-level autonomous underwater vehicle (AUV) missions and behaviors. A number of universities and laboratories are conducting AUV research and development that involves a wide variety of sensor types and sensor interpretation methods. The Defense Advanced Research Projects Agency (DARPA) Unmanned Undersea Vehicle (UUV) uses sidescan sonar and neural network classification for underwater mine detection [6]. Woods Hole Oceanographic Institution has used sidescan sonar, stochastic backprojection and a variety of vision processing techniques and sea floor shape information to create three-dimensional bottom images [7]. The University of New Hampshire Experimental Autonomous Vehicle (EAVE) III uses depth profiling, acoustic long baseline navigation and comparison with a world model to detect bottom objects [8]. Numerous other examples of sensor data interpretation exist. In contrast to most methods, this sonar classification system uses parametric regression, geometric analysis and expert system heuristics to create classifiable object types. An advantage of this approach is that progressively higher levels of abstraction are possible.

\section{OVERVIEW}

The objective of this paper is to present a method for autonomous classification of underwater objects. This is achieved using geometric sonar analysis techniques and an expert system for heuristic reasoning. Fig. 1 shows how low-level sonar data is processed to produce increasingly complex geometric objects and high-level classification outputs.

Geometric analysis can distill large amounts of sonar data into useful information that can be used to make logical and informed decisions. The primary difficulty in geometric sonar analysis is that active sonar signal returns are inherently noisy and unconnected. Parametric regression is a robust method of least-squares line fitting that permits precise geometric analysis of range and bearing data [9]. Generated regression lines are provided to a polyhedron-building algorithm to create geometric objects. Geometric object attributes can then be compared to known object types through the rule-based pattern-matching capabilities of an expert system, resulting in object classification.

The use of real world data is important for development and verification of a sonar classification expert system. Successful examples of expert system classifications using NPS AUV sonar data are described.

\section{GEOMETRIC ANALYSIS OF SONAR DATA}

\section{A. General Characteristics of Active Sonar Data}

Outputs common to practically all active sonars are range and bearing from the sonar transducer to a contact, if any is detected. Posture of an underwater vehicle includes a three-dimensional position coordinate, as well as vehicle attitude consisting of roll, elevation and azimuth orientations. The relative position of each sonar return is combined with vehicle posture using vector addition to yield a precise 


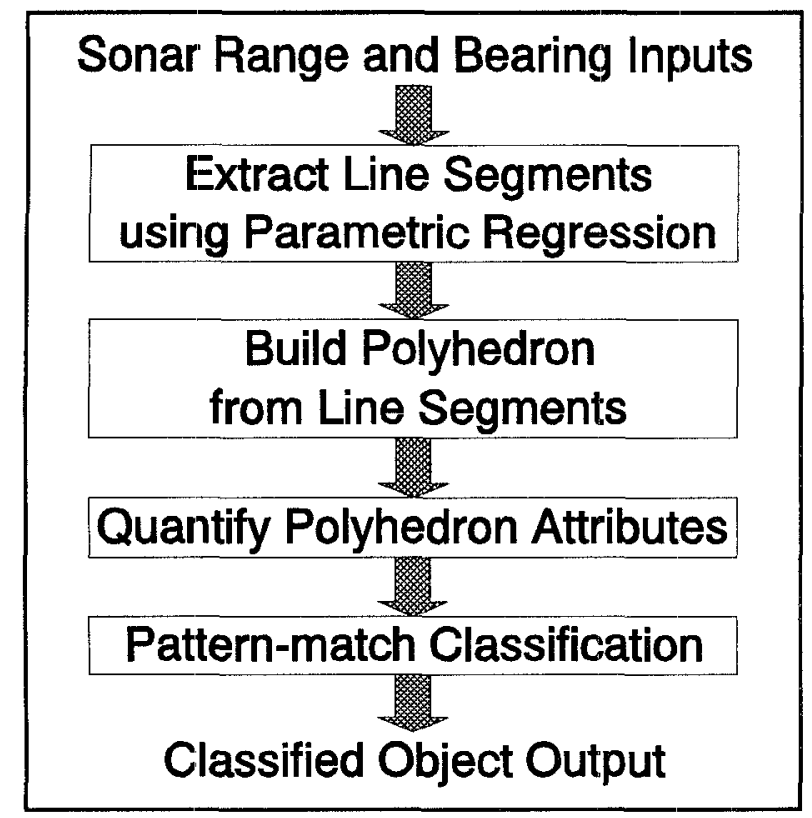

Fig. 1. Autonomous sonar classification process diagram.

three-dimensional coordinate. In this paper the term "sonar data" refers to simultaneous sonar range and bearing data returned from an active sonar transmission.

\section{B. Geometric Primitives and Object Attribute Definitions}

Sonar data can be analyzed to produce geometric forms such as points, lines or polyhedra. Precise definitions of geometric primitives and object attributes are necessary for predictable and repeatable sonar classifier performance. It is important that the theoretical basis of a sonar classification expert system be both mathematically rigorous and as general as possible in order to allow increasingly sophisticated analysis of data. A formal geometry-based approach also permits expert system compatibility with a wide variety of sonar types.

The geometric primitives considered by this expert system are point, line segment, polyhedron and cylindrical polyhedron (i.e. a three-dimensional polyhedron that extends vertically up and down from a planar polygon perimeter). Object attributes include centroid position, depth, length, width, height, perimeter, cross-sectional area, thinness, and volume. Indirect attributes such as positional accuracy, confidence factor, inferred edges and hidden edges are also evaluated. Additional geometric primitives and object attributes can be defined as necessary to utilize the more sophisticated data available from sector scanning, two-dimensional swath or three-dimensional multi-beam sonars. Similar approaches using curved shapes such as circles, ellipses or conics [5] are also compatible.

\section{Extracting Line Segments using Parametric Regression}

Linear relationships described by sets of discrete data are typically found using standard linear regression analysis, commonly known as least-squares fit. This method is widely used but has a significant limitation in that regression calculations on $(x, y)$ coordinate points parallel to the $y$-axis result in divide-by-zero singularities for slope and mathematically undefined regression results. Since typical unconstrained sonar data may lie on any orientation, a different method is needed for autonomous fitting of best-approximation line segments to discrete sonar returns.

The parametric regression method utilizes a polar coordinate derivation of linear regression analysis to provide a robust and accurate least-squares fit of line segments to sequences of data points. This method has been developed in detail and is particularly well suited for geometric analysis of real-world sonar data [9] [10] [11] [12]. Associated with each regression line segment is an elliptical thinness term that can be used as a metric for line segment accuracy and data variance. Fig. 2 shows a typical parametric regression line segment fit to a set of sonar returns.

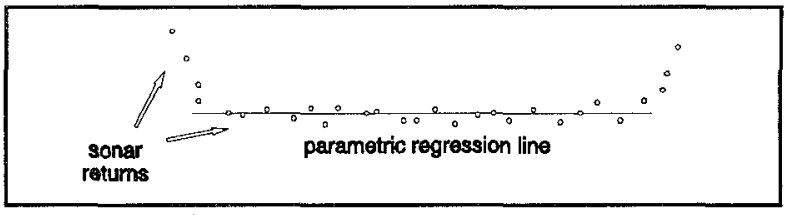

Fig. 2. Typical parametric regression line fit.

A further significant benefit of parametric regression analysis is that it is a sequential algorithm which provides immediate incremental improvements upon receipt of each individual data point. The sequential nature of this algorithm makes it highly suited for real-time operations that must meet immediate response requirements. Real-time vehicles cannot afford to wait for intermittently time-consuming sonar analysis when excessive delay might jeopardize navigational safety or mission performance.

\section{Building a Polyhedron from Line Segments}

Parametric regression provides linear one-dimensional geometric primitives. However line segments by themselves are insufficient for thorough two-dimensional spatial reasoning or object classification. A polyhedron-building algorithm is presented here as a means of constructing two-dimensional geometric objects from a sequence of regression line segments. In this context the polyhedron-building algorithm is a logical extension to the parametric regression algorithm.

One important assumption used when building polyhedra is that underwater contacts of interest have predominantly convex shapes, i.e. they contain no large concave depressions or cavities. This assumption permits clear delineation of independent object boundaries. Analysis of an actual concave object results in the definition of adjacent convex objects. Higher-level analysis at the heuristic level can be used to clump adjacent objects if needed.

Note that the orientation of vehicle sonar relative to detected objects is a critical consideration in the polyhedron-building algorithm, since spatial relationships are 
equally dependent on sensor perspective and actual object shape.

Polyhedron building begins with a single line segment produced by parametric regression analysis of continuous sonar data. Each following segment from regression analysis on the same sensor is compared to the previous segment. If the follow-on segment meets proximity and orientation criteria, then it is considered to be another part of the same geometric object. This segment comparison process is repeated until proximity or orientation criteria fail, at which time the previous geometric object is complete and the follow-on segment becomes the beginning segment of a new geometric object.

Proximity is measured between the end point of the most recently correlated line segment and the start point of the next segment to be considered. The proximity criterion is typically small and restrictive (e.g. less than 1 foot) in order to permit discrimination between adjacent objects. The proximity criterion must be met prior to comparing relative orientation for geometric object extension.

Orientation comparisons are made to determine whether adjacent segments are colinear, convex or concave. The colinear test allows a reasonable error bound (e.g. $\pm 10^{\circ}$ ) in order to account for sonar noise and line-fitting approximations. Colinear segments are acceptable for geometric object extension (Fig. 3).

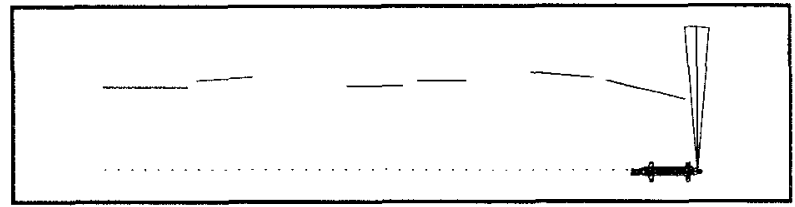

Fig. 3. Examples of colinear regression line segments.

The convex test measures whether the follow-on segment direction points farther away from the sensor's perspective than the previous segment. Convex segments are also acceptable for geometric object extension (Fig. 4).

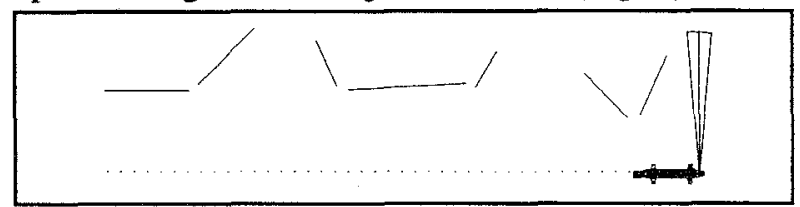

Fig. 4. Examples of convex regression line segments.

The concave test measures whether the follow-on segment direction points closer towards the sensor's perspective than the previous segment, in effect defining the boundaries of a hole. Concave line segment relative orientations indicate a break between separate convex geometric objects (Fig. 5). The follow-on segment is used to start a new polyhedron.

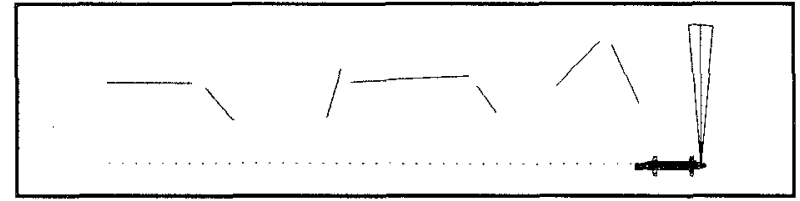

Fig. 5. Examples of concave regression line segments.
Inferred edges are presumed to exist between each pair of the sequential detected edges that make up a polyhedron. A single hidden edge is presumed to exist between the start point and end point of a particular object. The classifier must recognize, however, that such hidden edges may be completely inaccurate since the actual hidden sides of the object were obscured from the sonar. Fig. 6 illustrates the polyhedron-building algorithm.

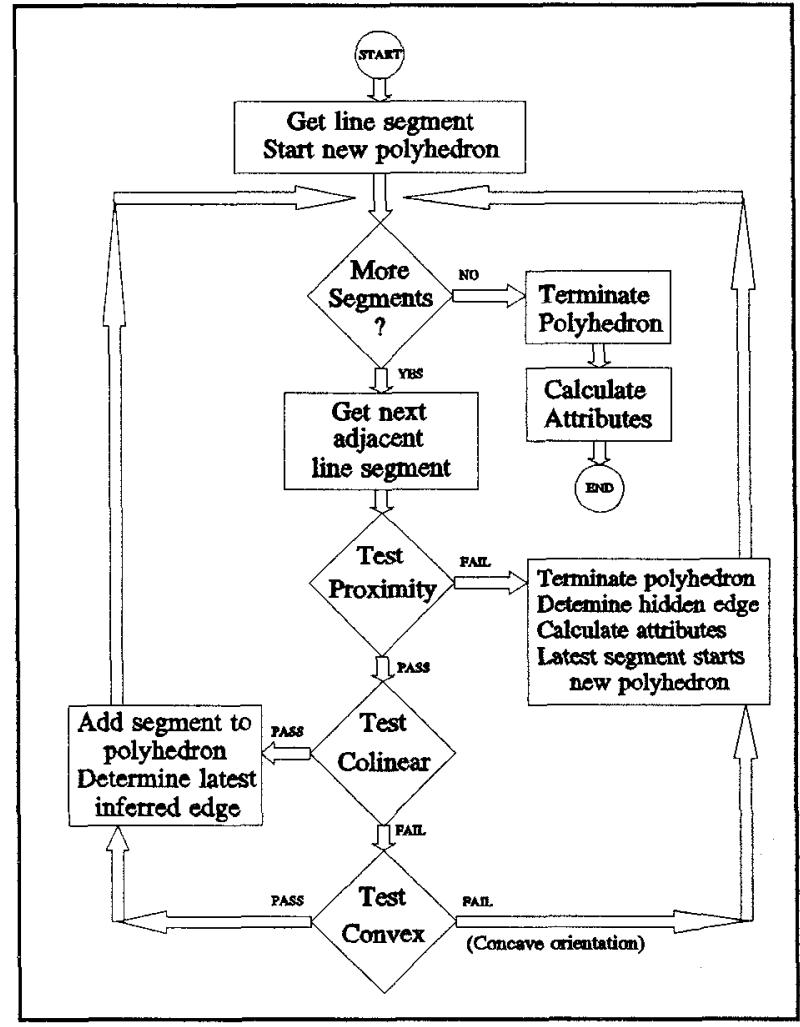

Fig. 6. Algorithm to build polyhedra from line segments.

\section{E. Quantifying Polyhedron Attributes}

The attributes that are used to classify objects need to be precisely defined and calculated, wherever possible. For example, attributes such as depth, length, width and height are directly measurable using calculated sonar positions. Object perimeter can be determined by first summing the lengths of all correlated line segments, and then adding the lengths of all inferred and hidden edges that are presumed to exist between detected edges. Fig. 7 shows how the start point, regression line segments, inferred edges and hidden edge that make up a polyhedron cross-section define a series of triangular areas.

Area of a single planar triangle is given by (1).

$$
\text { Area }_{\Delta}=\frac{1}{2}\left|\left(X_{2}-X_{1}\right)\left(Y_{3}-Y_{1}\right)-\left(X_{3}-X_{1}\right)\left(Y_{2}-Y_{1}\right)\right|
$$

Polyhedron cross-section area is determined by summing the area of these triangles, given by (2).

Centroid position for a planar triangle is calculated by averaging the respective $\mathrm{X}$ and $\mathrm{Y}$ coordinates of each vertex. 


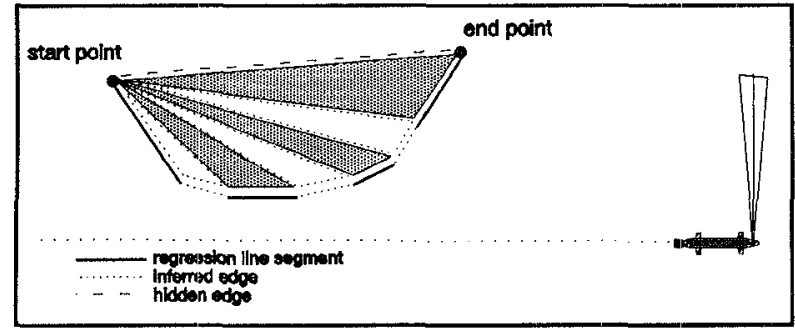

Fig. 7. Summing triangle areas to determine polyhedron cross-sectional area.

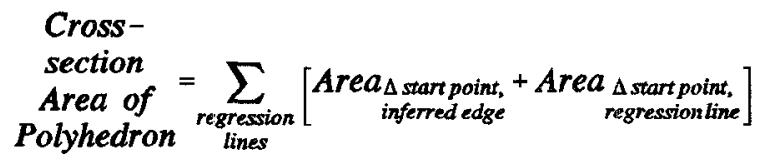

Centroid position for the polyhedron cross-section is precisely deternined by taking the weighted average of each of the triangle centroids, given by (3).

$$
\begin{gathered}
\begin{array}{c}
\text { Polyhedron } \\
\text { Cross-section } \\
\text { Centroid }
\end{array}=\left(\begin{array}{l}
\frac{\text { Area }_{\Delta_{1}} X_{C_{1}}+\cdots+\text { Area }_{\Delta_{N}} X_{C_{N}}}{\text { Area }_{\text {Polyhedron }}}, \\
\frac{\text { Area }_{\Delta_{1}} Y_{C_{1}}+\cdots+\text { Area }_{\Delta_{N}} Y_{C_{N}}}{\text { Area }}{ }_{\text {Polyhedron }}
\end{array}\right)
\end{gathered}
$$

Polyhedron cross-section thinness is a useful attribute for classification. It is defined as the ratio of polyhedron cross-section area to the square of polyhedron perimeter.

If object height is needed and has not been directly measured by sonar data, it can be estimated using heuristic rules based on object depth, bottom depth or independent object classification. Object volume is the product of cross-sectional area and measured or estimated object height.

Indirect attributes such as positional accuracy, confidence factor, inferred edges and hidden edges are also evaluated. Point positional accuracy is derived by combining current vehicle positional accuracy estimate with sonar accuracy or sonar beamwidth at the range to the object. Confidence factor can be defined independently of positional accuracy as a measure of how well the object matches a classification rule. Hidden edge length is a measure of what is not known about the object. Defining initial classification confidence factor as the ratio between hidden edge length and detected perimeter indicates how much of the contact has actually been evaluated. Hidden edge metrics can be used to indicate whether further sonar investigation of the contact is desirable. Fig. 8 shows detected edges, inferred edges and hidden edge relative to processed sonar returns, and how these geometric primitives may not fully reveal all features of a contact.

IV. EXPERT SYSTEM HEURISTICS FOR SONAR CLASSIFICATION

While geometric analysis can be defined with mathematical precision, human knowledge regarding sonar classification is less rigorous and can be encoded as expert system heuristics.

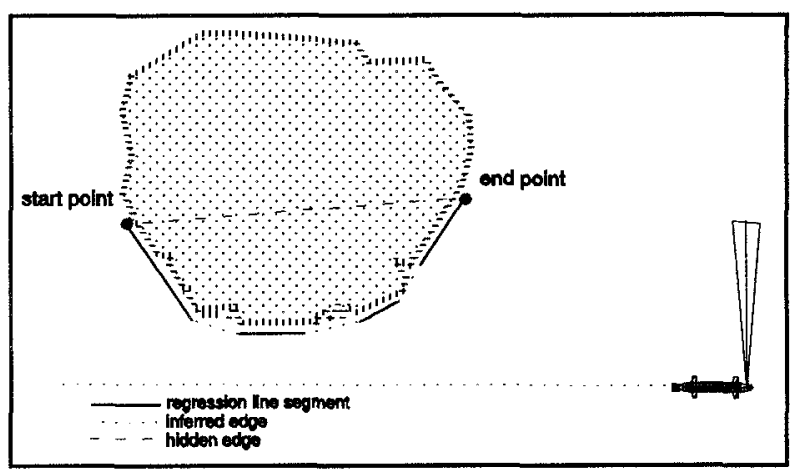

Fig. 8. Polyhedron detected edges, inferred edges and hidden edge may not fully reveal all features of the sonar contact.

\section{A. Classification Heuristics and Attribute Heuristics}

Sonar classification is not always a well defined problem. For example, it is possible that sonar analysis of a single object can be performed from different perspectives and lead to completely different classifications. An analogy to classifying objects using simple range and bearing sonars is attempting to identify your surroundings while looking at the world through a steerable pinhole. It is difficult! Consequently, sonar classification criteria are often ambiguous and difficult to quantify, even when using formally derived geometric primitives. However, the heuristic approach used by expert systems is effective in many types of inexact problems and enables an autonomous system to obtain excellent sonar classification results.

Heuristics can be used for evaluating attributes such as object height when information is incomplete. Both attribute and classification heuristics can be easily modified in understandable ways despite the ambiguities of sonar analysis. The intuitive power of heuristics combined with the precision of geometric analysis gives sonar classification expert systems wide applicability and adaptability.

For this expert system approach, classification of sonar contacts is performed by comparing attributes of detected objects with predetermined attributes of known objects of interest. Different classification criteria are necessary and desirable for different environments. In particular, the different characteristics of deep ocean versus shallow water versus an artificial pool will constrain the possible types of objects to be detected. Knowledge of the current environment can be extremely useful when determining the specialized classification rules and heuristic criteria to be used for a given mission.

Precise classification of every possible object type may not be necessary for some missions. Resolution of an ambiguous classification typically requires multiple sensor looks, costing additional time and energy. Preliminary classification as a potential contact of interest may be sufficient to justify maneuvering for additional sensing and closer investigation. Conversely, objects deemed to be of no interest require no further investigation by the vehicle. 
Size can be the primary classification attribute for most underwater objects of interest. However, size per se is not a strictly defined term. It is worth mention that significant object size may be indicated by a variety of attributes including cross-sectional area, volume, perimeter, thinness or hidden edge length. Any or all of these size-related attributes may require close evaluation in order to properly discriminate between similarly-sized sonar targets such as mines and rocks.

\section{B. Pattern-match Classification Examples}

Examples of how heuristic rules work can illustrate how a sonar expert system can classify objects. Two examples are presented here.

Preliminary wall classification is possible during the execution of the polyhedron-building algorithm. Walls are defined as any flat linear surface of non-trivial length. Polyhedra being built can be considered walls as long as each of the newly added regression line segments meet colinearity and proximity criteria. As soon as the polyhedron-building algorithm adds a new line segment based on convexity criteria, the polyhedron being built can be immediately reclassified from wall to object since the polyhedron is no longer linear.

Once a polyhedron has been built, all polyhedron attributes are automatically calculated. At this final stage, all of the preliminary work to quantitatively determine precise geometric objects greatly simplifies object classification. For instance, a polyhedron might be classified as a mine-like object whenever cross-sectional area is between 10 and 100 square feet. Other classifications are equally straightforward.

Some objects should not be uniquely classified. For example, discrimination between a scuba diver and a mine-like object may be difficult. A particular strength of the expert system approach is that each object can receive multiple classifications with associated confidence factors as appropriate. This feature allows high-level reasoning using uncertainty, rather than being constrained by an arbitrary and potentially incorrect single classification.

What was originally an intractable sonar classification problem is now much simpler and understandable at the highest level of the expert system.

\section{IMPLEMENTATION AND EVALUATION}

A. NPS AUV Vehicle Description and Sonar Characteristics

Naval officers and civilian scientists at NPS are conducting active research using an AUV designed and constructed at the school. The NPS AUV is used for basic research and thesis work in control systems technology, artificial intelligence, scientific visualization and systems integration. Specific NPS AUV project objectives include the study of mission planning, navigation, collision avoidance, real-time mission control, replanning, object recognition, vehicle dynamic motion control, and post-mission data analysis [15] [16] [17]. Video clips showing in-water NPS AUV operation are available in [17] [18].

\section{B. CLIPS Expert System}

CLIPS ("C" Language Integrated Production System) was chosen for this application due to its portability, extendability, capabilities, thorough documentation and interactive tutorials [19] [20]. Since it is written in "C", CLIPS can run under most computer architectures. CLIPS support includes an active user base, annual applications conferences, an applications abstract registry and complete source code [21]. C. NPS AUV Integrated Simulator

Typically the development and testing of AUV hardware and software is greatly complicated by vehicle inaccessibility during operation. Integrated simulation remotely links vehicle components and support equipment with graphics simulation workstations. Integration of actual AUV components with three-dimensional simulation allows complete real-time, pre-mission, pseudo-mission and post-mission visualization and analysis in the lab. Integrated simulator testing of AUVs is a broad and versatile method that was essential in the development of the NPS AUV sonar classification expert system [17] [22].

\section{EXPERIMENTAL RESULTS}

An example best demonstrates successful classification of actual sonar returns. The NPS AUV was programmed to follow a racetrack traversal of the pool and record all pertinent data. A single swimmer represented a mine-like object near the right-hand wall of the NPS swimming pool, shown in Fig. 9. Individual left transducer sonar returns are plotted as small circles and vehicle track as a large oval in Fig. 10, while the line segments calculated by the parametric regression algorithm are shown superimposed over corresponding sonar data. Small distortions are evident due to unmodeled sideslip navigational error in vehicle track data.

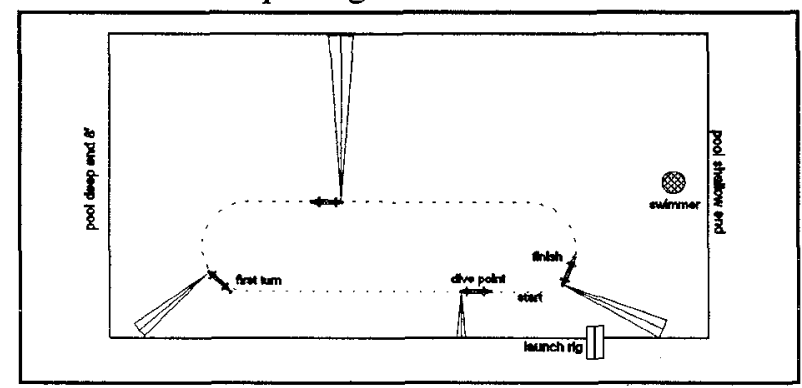

Fig. 9. NPS AUV test track using left transducer only.

Note swimmer target.

Sonar data recorded by the NPS AUV in the pool are uploaded after mission completion via modem and processed off-line by the authors' sonar classification expert system. Classification results are then graphically rendered by the NPS AUV Integrated Simulator running on a Silicon Graphics Iris workstation. This three-dimensional display shows all generated parametric regression line segments, inferred edges, hidden edges, and detected walls. Using the same data as in Fig. 10, the three-dimensional graphics display as seen from a viewpoint above the pool is shown in Fig. 11. The target of interest met classification criteria for a 


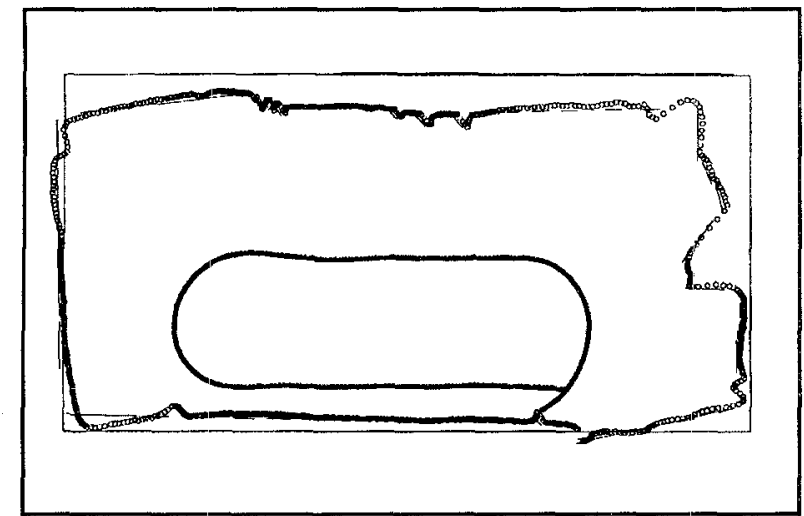

Fig. 10. NPS AUV sonar classification expert system plot of pool data and parametric regression line segments.

mine-like object and a simulator closeup is shown in Fig. 12. The authors' assessment of experimental results is that the NPS AUV Autonomous Sonar Classification System is highly effective at classifying objects.

\section{ACKNOWLEDGMENTS}

The authors wish to thank CDR Charles A. Floyd USN for his assistance in implementing parametric regression, Russell Whalen for underwater photography and test support, Dr. Neil C. Rowe and Dr. Michael J. Zyda for critical analysis, and Dr. Anthony M. Healey and Dr. Robert B. McGhee for continuing guidance and inspiration.

This paper was considerably edited to fit conference space requirements. Complete copies are available upon request.

\section{REFERENCES}

[1] Hebert, Martial, Kanade, Takeo and Kweon, InSo, "3-D Vision Techniques for Autonomous Vehicles," NSF Range Image Understanding Workshop, 1988, pp. 273-337.

[2] Besl, P.J. and Jain, R.C., "Three-Dimensional Object Recognition," Computing Surveys, vol. 17 no. 1, March 1985, pp. 77-145.

[3] Iyengar, S. Sitharama and Elfes, Alberto, ed., Autonomous Underwater Robots: Perception, Mapping and Navigation, volume 1, IEEE Computer Society Press, Los Alamitos, Califomia, 1991.

[4] Luo, R.C. and Kay, M.G., "Multisensor Integration and Fusion in Intelligent Systems," IEEE Transactions on Systems, Man and Cybernetics, vol. 19 no. 5, September/October 1989, pp. 901-931.

[5] Moravec, Hans, "The Stanford Cart and the CMU Rover," Proceedings of the IEEE, vol. 71 ro. 7 , July 1983 , pp. 872-884.

[6] Pappas, George, Shotts, William, O'Brien, Mack and Wyman, William, "The DARPA/Navy Unmanned Undersea Vehicle Program," Unmanned Systems, vol. 9 no. 2, Spring 1991, pp. 24-30.

[7] Stewart, W. Kenneth, "Three-DimensionalModeling of Seafloor Backscatter from Sidescan Sonar for Autonomous Classification and Navigation, "Proceedings of the 6th Intemational Symposium on Unmanned Untethered Submersible Technology, University of New Hampshire, Durham, New Hampishire, June 1989, pp. 372-392.

[8] Blidberg, D.R., Chappell, S., Jalbert, J., Turner, R., Sedor, G. and Eaton, P., "The EAVE AUV Program at the Marine Systems Engineering Laboratory," Proceedings of 1st LARP Workshop on Mobile Robots for Subsea Environments, Monterey, California, October 1990, pp. 33-42.

[9] Floyd, Charles A., Design and Implementation of a Collision Avoidance System for the NPS Autonomous Underwater Vehicle (AUVII) Utilizing Ultrasonic Sensors, Master's Thesis, Naval Postgraduate School, Monterey, California, September 1991

[10] Kanayama, Yutaka and Noguchi, Tetsuo, "Spatial Learning by an Autonomous Mobile Robot with Ultrasonic Sensors, "University of California Santa Barbara Department of Computer Science Technical Report TRCS89-06, February 1989.

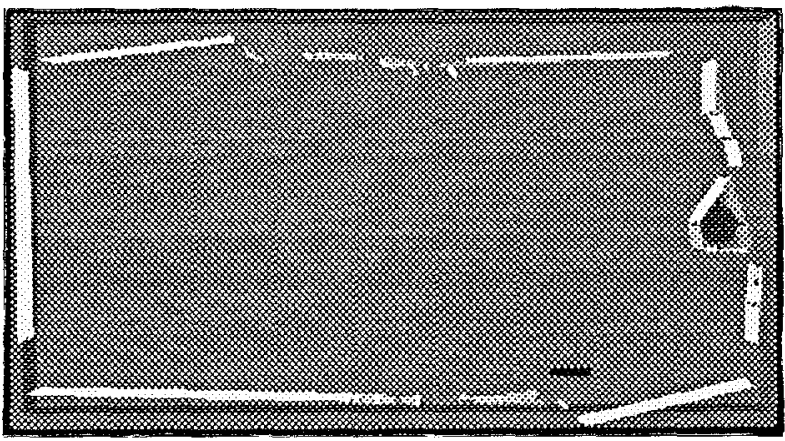

Fig. 11. Integrated simulator screen display of the full NPS pool and all sonar classifications.

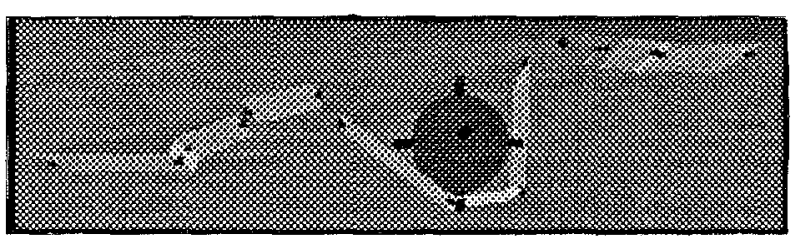

Fig. 12. Integrated simulator display close-up of a mine-like object classified by the sonar expert system using detected edges, inferred edges, hidden edge and cross-sectional area.

[11] Kanayama, Yutaka, Noguchi, Tetsuo, and Hartman, Bruce, "Sonar Data Interpretation for Autonomous Mobile Robots," unpublished paper, Naval Postgraduate School, Monterey, California, 1990.

[12] Floyd, Charles A., Kanayama, Yutaka, and Magrino, Christopher, "Underwater Obstacle Recognition using a Low-Resolution Sonar," Proceedings of the Seventh International Symposium on Unmanned Untethered Submersible Technology, University of New Hampshire, Durham, New Hampshire, September 1991, pp. 309-327.

[13] Jackson, Peter, Introduction to Expert Systems, Addison-Wesley Publishing Co. Inc., Workingham, England, 1991.

[14] Sacerdoti, Earl D., "Managing Expert System Development," AI Expert, vol. 6 no. 5, May 1991, pp. 26-33.

[15] Healey, A.J., McGhee, R.B., Christi, R., Papoulias, F.A., Kwak, S.H., Kanayama, Y. and Lee, Y., "Mission Planning, Execution and Data Analysis for the NPS AUV II Autonomous Underwater Vehicle," Proceedings of Ist IARP Workshop on Mobile Robots for Subsea Environments, Monterey, California, October 1990, pp. 177-186.

[16] Brutzman, Donald P. and Compton, Mark A., "AUV Research at the Naval Postgraduate School," Sea Technology, vol. 32 no. 12, December 1991, pp. 35-40.

[17] Brutzman, Donald P., NPS AUV Integrated Simulator, Master's Thesis, Naval Postgraduate School, Monterey, California, March 1992.

[18] Floyd, Charles A., Brutzman, Donald P. and Whalen, Russell, "Naval Postgraduate School Autonomous Underwater Vehicle," Video Proceedings of the IEEE International Conference on Robotics and Automation 92, Nice, France, May 1992.

[19] Giarratano, Joseph C., CLIPS User's Guide, NASA, Lyndon B. Johnson Space Center, January 1991.

[20] NASA Software Technology Branch, CLIPS Reference Manual, Lyndon B. Johnson Space Center, Houston, Texas, 1991.

[21] Brooke, Tom, "The Art of Production Systems," AI Expert, vol. 7 no. 1, January 1992, pp. 30-35.

[22] Brutzman, Donald P., "Integrated Simulation for Rapid Development of Autonomous UnderwaterVehicles", Proceedings of the IEEE Oceanic Engineering Society Conference AUV 92, Washington DC, June 1992.

[23] Corkill, Daniel, "Blackboard Systems," AI Expert, vol. 6 no. 9, September 1991, pp. $40-47$. 